\title{
NOTE ON THE SUPPLEMENT TO THE LAW OF CUBIC RECIPROCITY
}

\author{
KENNETH S. WILLIAMS ${ }^{1}$
}

ABSTRACT. A short proof is given of the supplement to the law of cubic reciprocity proved by Eisenstein in 1844.

Let $\omega=(-1+\sqrt{ }-3) / 2$. Let $\pi=a+b \omega$ be a primary complex prime in the Eisenste in domain $Z[\omega]$ so that

$$
a \equiv 2(\bmod 3), \quad b \equiv 0(\bmod 3)
$$

say

$$
a=3 m-1, \quad b=3 n,
$$

and

$$
a^{2}-a b+b^{2}=\pi \bar{\pi}=p,
$$

where $p$ is a rational prime $\equiv 1(\bmod 3)$ (see for example [3, Chapter 9]). The cubic residue character $(\cdot / \pi)_{3}$ modulo $\pi$ is defined by

$$
(\alpha / \pi)_{3}=\omega^{r} \text { if } \alpha^{(p-1) / 3} \equiv \omega^{r}(\bmod \pi),
$$

where $r=0,1,2$ and $\alpha \in Z[\omega]$ is such that $\alpha \neq 0(\bmod \pi)$. The supplement to the law of cubic reciprocity proved by Eisenste in [2] in 1844 states that

$$
((1-\omega) / \pi)_{3}=\omega^{2 m} \text {. }
$$

We remark that $1-\omega$ is a prime factor of 3 in $Z[\omega]$. Here is a simple proof of this result (see comment in [3, p. 115])。

Let $(h, k)_{3}$ denote the number of solutions $(r, s)$ of $1+g^{3 r+h} \equiv$ $g^{3 s+k}(\bmod p)$, with $0 \leq r, s<(p-1) / 3$, where $g$ is a primitive root $(\bmod p)$ such that $(g / \pi)_{3}=\omega$. (If $g$ is such that $(g / \pi)_{3}=\omega^{2}$, we can replace $g$ by

Received by the editors January 31, 1974 .

AMS (MOS) subject classifications (1970). Primary 10A15; Secondary 12C20.

Key words and phrases. Supplement to law of cubic reciprocity, cubic residue character, Eisenstein domain.

${ }^{1}$ Research supported by National Research Council of Canada grant no. A-7233. 
an appropriate power of $g$ so that this power of $g$ is a primitive root with cubic residue character $(\bmod \pi)$ equal to $\omega_{\text {. }}$ ) Then it is well known (see for example [1, p. 397]) that

$$
\begin{aligned}
& 9(0,0)_{3}=p-8+2 a-b, \\
& 9(0,1)_{3}=9(1,0)_{3}=9(2,2)_{3}=p-2-a+2 b, \\
& 9(0,2)_{3}=9(2,0)_{3}=9(1,1)_{3}=p-2-a-b, \\
& 9(1,2)_{3}=9(2,1)_{3}=p+1+2 a-b .
\end{aligned}
$$

From the work of Muskat [4, Corollary 1 with $e=3]$ we have

$$
\text { ind }_{g}(3) \equiv(1,1)_{3}-(2,2)_{3}(\bmod 3) \text {, }
$$

where, for any integer $a \not \equiv 0(\bmod p)$, ind ${ }_{g}(a)$ denotes the unique integer $b$ such that $a \equiv g^{b}(\bmod p), 0 \leq b \leq p-2$. Putting (2), (6) and (7) together we obtain

$$
\text { ind }_{g}(3) \equiv-n(\bmod 3) \text {, }
$$

So that $3^{(p-1) / 3} \equiv g^{-n(p-1) / 3} \equiv \omega^{-n}(\bmod \pi)$, showing that $(3 / \pi)_{3}=\omega^{-n}$. Hence

$$
\left(\frac{1-\omega}{\pi}\right)_{3}=\left(\frac{(1-\omega)^{4}}{\pi}\right)_{3}=\left(\frac{3^{2} \omega^{2}}{\pi}\right)_{3}=\omega^{2(p-1) / 3-2 n},
$$

and the result follows since from (2) and (3) we have $(p-1) / 3 \equiv m+n$ $(\bmod 3)$.

\section{REFERENCES}

1. L. E. Dickson, Cyclotomy, higher congruences and Waring's problem, Amer. J. Math. 57 (1935), 391-424.

2. G. Eisenstein, Nachtrag zum kubischer Reciprocitätssatze, J. Reine Angew. Math. 28 (1844), 28-35.

3. K. Ireland and M. I. Rosen, Elements of number theory, Bogden and Quigley, Tarrytown-on-Hudson, New York, 1972.

4. J. B. Muskat, On the solvability of $x^{e} \equiv e(\bmod p)$, Pacific J. Math. 14(1964), 257-260. MR 28 \#2997.

DE PARTMENT OF MATHEMATICS, CARLETON UNIVERSITY, OTTAWA, ONTARIO, CANADA 